\title{
BMJ Open Can we IMPROVE cardiovascular outcomes through phosphate lowering in CKD? Rationale and protocol for the IMpact of Phosphate Reduction On Vascular End-points in Chronic Kidney Disease (IMPROVE-CKD) study
}

Nicole Lioufas, ${ }^{1,2}$ Nigel D Toussaint, ${ }^{1,2}$ Eugenia Pedagogos, ${ }^{3}$ Grahame Elder, ${ }^{4}$ Sunil V Badve, ${ }^{5}$ Elaine Pascoe,,${ }^{6,7}$ Andrea Valks, ${ }^{8}$ Carmel Hawley, ${ }^{6,7}$ on behalf of the IMPROVE-CKD Writing Committee.

To cite: Lioufas N, Toussaint ND, Pedagogos E, et al. Can we IMPROVE cardiovascular outcomes through phosphate lowering in CKD? Rationale and protocol for the IMpact of Phosphate Reduction On Vascular End-points in Chronic Kidney Disease (IMPROVECKD) study. BMJ Open 2019;9:e024382. doi:10.1136/ bmjopen-2018-024382

- Prepublication history and additional material for this paper are available online. To view these files, please visit the journal online (http://dx.doi. org/10.1136/bmjopen-2018024382).

Received 24 May 2018 Revised 9 October 2018 Accepted 8 January 2019

D Check for updates

(C) Author(s) (or their employer(s)) 2019. Re-use permitted under CC BY-NC. No commercial re-use. See rights and permissions. Published by BMJ.

For numbered affiliations see end of article.

Correspondence to

Nigel D Toussaint;

nigel.toussaint@mh.org.au

\section{ABSTRACT}

Introduction Patients with chronic kidney disease (CKD) are at heightened cardiovascular risk, which has been associated with abnormalities of bone and mineral metabolism. A deeper understanding of these abnormalities should facilitate improved treatment strategies and patient-level outcomes, but at present there are few large, randomised controlled clinical trials to guide management. Positive associations between serum phosphate and fibroblast growth factor 23 (FGF-23) and cardiovascular morbidity and mortality in both the general and CKD populations have resulted in clinical guidelines suggesting that serum phosphate be targeted towards the normal range, although few randomised and placebocontrolled studies have addressed clinical outcomes using interventions to improve phosphate control. Early preventive measures to reduce the development and progression of vascular calcification, left ventricular hypertrophy and arterial stiffness are crucial in patients with CKD.

Methods and analysis We outline the rationale and protocol for an international, multicentre, randomised parallel-group trial assessing the impact of the noncalcium-based phosphate binder, lanthanum carbonate, compared with placebo on surrogate markers of cardiovascular disease in a predialysis CKD populationthe IMpact of Phosphate Reduction On Vascular Endpoints (IMPROVE)-CKD study. The primary objective of the IMPROVE-CKD study is to determine if the use of lanthanum carbonate reduces the burden of cardiovascular disease in patients with CKD stages $3 \mathrm{~b}$ and 4 when compared with placebo. The primary end-point of the study is change in arterial compliance measured by pulse wave velocity over a 96-week period. Secondary outcomes include change in aortic calcification and biochemical parameters of serum phosphate, parathyroid hormone and FGF-23 levels.

Ethics and dissemination Ethical approval for the IMPROVE-CKD trial was obtained by each local Institutional Ethics Committee for all 17 participating sites in Australia,
Strengths and limitations of this study

- Largest cohort and longest in duration for randomised, placebo-controlled trial of phosphate binders in non-dialysis chronic kidney disease (CKD) population.

- Assesses potential effects, yet to be determined, of phosphate lowering on arterial compliance and vascular calcification in patients with CKD.

- Avoids exogenous calcium load by using non-calcium-based phosphate binders in blinded fashion to inform clinical guidelines lacking strong evidence for phosphate control.

- Will provide valuable information regarding relationship between phosphate, fibroblast growth factor 23 and arterial compliance and calcification in CKD.

- Limitations include potential difficulty in recruitment of large CKD population to assess thrice-daily phosphate binders, and possible lack of adherence to study medication over a 96-week period.

New Zealand and Malaysia prior to study commencement. Results of this clinical trial will be published in peerreviewed journals and presented at conferences.

Trial registration number ACTRN12610000650099.

\section{INTRODUCTION}

'Chronic kidney disease-mineral bone disorder' (CKD-MBD) has been accepted terminology in nephrology research and clinical practice for more than a decade. This clinical entity encompasses intimately related abnormalities of mineral homeostasis, bone turnover and mineralisation, and vascular and soft tissue calcification, which are almost universal in patients with advanced CKD. CKD-MBD is a complex and 
evolving area for which international clinical guidelines have been published to provide suggestions and recommendations on management. ${ }^{1}$ Just as importantly, these guidelines highlight the paucity of clinical studies supporting suggested biochemical targets, and the lack of evidence-based treatment strategies aimed at reducing the risks of fracture, cardiovascular disease and mortality or improving quality of life for individuals with CKD.

Patients with CKD have significantly increased risks of cardiovascular disease and all-cause mortality compared with the general population, ${ }^{2}$ and traditional and CKD-specific risk factors contribute to this risk. ${ }^{3}$ Abnormalities of bone and mineral metabolism, and in particular abnormal values of serum phosphate, calcium, parathyroid hormone (PTH) and fibroblast growth factor 23 (FGF-23), are considered important; however, few strong recommendations for treatment of these values exist beyond normalising serum phosphate and lowering PTH to an indeterminate target. ${ }^{1}$ Minimising exogenous calcium through reducing exposure to calcium-based phosphate binders within the CKD population has also been suggested as beneficial, citing meta-analyses showing mortality benefits of non-calcium containing phosphate binders over calcium-based binders, ${ }^{45}$ but placebo-controlled studies are lacking.

While observational studies show associations between higher serum phosphate levels and worse clinical outcomes, causality has never been demonstrated. Serum phosphate provides a poor measure of overall phosphate balance or the risk of soft tissue calcification in patients with CKD, and benefits of phosphate lowering are yet to be demonstrated. Whether a target level of serum phosphate will assist in attaining improved outcomes remains controversial and no randomised controlled trial (RCT) to date has proved that lowering extracellular phosphate improves clinical outcomes. The IMPROVE-CKD (IMpact of Phosphate Reduction On Vascular Endpoints in Chronic Kidney Disease) study is a placebo-controlled RCT assessing the potential effects of lanthanum carbonate, a non-calcium-based phosphate binder, on surrogate markers of cardiovascular disease in patients with CKD. Here, we discuss the rationale and study design of this important trial.

\section{Rationale for a clinical trial}

Epidemiological studies in the general population and in patients with CKD have consistently shown that serum phosphate levels within and above the upper normal range are independently and positively correlated with all-cause mortality. ${ }^{6}$ In patients with CKD, cardiovascular mortality, arterial stiffness and progression of renal impairment have also been correlated with higher serum phosphate. ${ }^{78}$ Positive phosphate balance is known to contribute to rising PTH and FGF-23 values, which in turn, have also been associated with adverse clinical outcomes. ${ }^{910}$ Thus, it is biologically plausible that therapeutic strategies aimed at reducing phosphate balance and serum phosphate values in patients with CKD might be beneficial. Dietary phosphate absorption is one modifiable determinant of phosphate balance and pilot studies that have evaluated phosphate binders, low phosphate diet or nicotinamide (which inhibits intestinal sodium-dependent phosphate cotransport) in patients with CKD have shown a reduction of dietary phosphate absorption, serum phosphate and FGF-23 levels. ${ }^{11}$ However, evidence for the benefit of such strategies on surrogates for cardiovascular disease or for CKD progression remains elusive.

Abnormal mineral metabolism, with alterations in the regulation of calcium, phosphate and FGF-23, is also associated with the development of vascular calcification. ${ }^{12}$ Although mechanisms leading to vascular calcification are incompletely understood, hypotheses include a maladaptive repair response to vascular and endothelial damage, ${ }^{13}$ and enhancement of concentration-dependent calcium-phosphate precipitation and extracellular matrix deposition due to elevated phosphate levels. Studies also report coronary artery calcification associated with elevated FGF-23 values independent of serum phosphate, ${ }^{10}{ }^{14}$ although this association is not a consistent finding. ${ }^{15}$ In addition to abnormalities of mineral metabolism, exogenous calcium has been associated with the development and progression of vascular calcification in patients with CKD. A meta-analysis of phosphate binders by Jamal et al, analysing 14 RCTs, reported a reduction in mortality associated with the use of non-calcium containing phosphate binders. ${ }^{4} \mathrm{~A}$ more recent meta-analysis of 25 eligible studies showed a reduction in all-cause mortality with sevelamer versus calcium-based binders. ${ }^{5}$ This difference is potentially related to a positive calcium balance in patients prescribed calcium-based phosphate binders leading to increasing vascular calcification. Another meta-analysis reviewed the individual contribution of available non-calcium-based phosphate binders, and reported that current data are more robust for sevelamer than for lanthanum or iron-based binders. ${ }^{16}$

The recently updated Kidney Disease Improving Global Outcomes (KDIGO) CKD-MBD Guidelines ${ }^{17}$ highlighted the lack of trial data demonstrating that lowering serum phosphate improved patient-centred outcomes. In fact, the evidence for any phosphate-lowering intervention is only based on epidemiological studies and biological plausibility. Although several recent historical cohort analyses (DOPPS, ArMORR and COSMOS) suggested that the prescription of phosphate binders to dialysis patients improved survival, ${ }^{18-20}$ these reports were not included in the evidence review for the updated KDIGO Guidelines. However, a recent RCT comparing calcium and non-calcium-based phosphate binders with placebo in 148 patients with CKD stages $3-4^{21}$ was included in the guideline update review and reported a minimal reduction of serum phosphate in the active treatment groups, no overall effect on FGF-23 and increases in coronary artery calcification for the combined phosphate binder groups (predominantly associated with the calcium-based binder arm). 
Table 1 Placebo-controlled randomised controlled trails (RCTs) of phosphate lowering in patients with CKD stages 3-5 not on dialysis assessing surrogate parameters of cardiovascular disease

\begin{tabular}{|c|c|c|c|}
\hline Study & Population & Intervention & Finding \\
\hline $\begin{array}{l}\text { Chue et al } \\
\text { (CRIB-PHOS) }^{22}\end{array}$ & $\begin{array}{l}120 \text { patients CKD3 non- } \\
\text { diabetic }\end{array}$ & Sevelamer carbonate vs placebo & $\begin{array}{l}\text { Lower urinary phosphate and FGF-23 (latter in } \\
\text { compliant patients) }\end{array}$ \\
\hline Seifert et $a l^{23}$ & 38 patients CKD3 & Lanthanum vs placebo & $\begin{array}{l}\text { No differences in serum/urine phosphate, PWV, } \\
\text { carotid IMT, vascular calcification }\end{array}$ \\
\hline IMPROVE-CKD & $\begin{array}{l}\text { Aimed recruitment of } 488 \\
\text { patients with CKD3b-4 }\end{array}$ & Lanthanum vs placebo & Ongoing \\
\hline
\end{tabular}

CKD, chronic kidney disease; COMBINE, CKD Optimal Management With BInders and NicotinamidE study; CRIB-PHOS, Chronic Renal Impairment in Birmingham- Phosphate study; FGF-23, fibroblast growth factor 23; IMPROVE-CKD, Impact of Phosphate Reduction On Vascular End-points in CKD; IMT, intimal medial thickness; PNT, phosphate normalisation trial; PWV, pulse wave velocity.

Similarly, two placebo-controlled RCTs of phosphate-lowering in predialysis CKD have also failed to support the benefit of phosphate lowering in this population (table 1). Chue et al found no significant differences between sevelamer and placebo with regard to left ventricular mass, systolic and diastolic function or pulse wave velocity (PWV) in 109 patients with CKD after 40 weeks, although only $56 \%$ of subjects took $\geq 80 \%$ of the prescribed therapy. ${ }^{22}$ In the adherent subgroup, treatment with sevelamer was associated with lower urinary excretion of phosphate and serum FGF-23, although binder use was not associated with cardiovascular outcomes of interest such as left ventricular hypertrophy or arterial compliance. In a smaller study of 38 CKD patients, Seifert et al reported no differences between lanthanum or placebo over 12 months with respect to serum or urinary phosphate, or surrogate cardiovascular markers of PWV, carotid artery intima-media thickness or vascular calcification. ${ }^{23}$ These results question the efficacy and safety of phosphate binders in this population and the updated KDIGO guidelines have amended a previous recommendation statement to maintain serum phosphate in the normal range for patients with CKD stages 3-5 to now suggest that treatment should focus on patients with overt hyperphosphatemia, although this recommendation is still based on weak clinical evidence.

Currently, there are two relevant RCTs in progress (table 1). The COMBINE (CKD Optimal Management With BInders and NicotinamidE) study assesses the effects of lowering serum phosphate and FGF-23 levels in patients with CKD stages $3-4 .^{11}$ This study aims to recruit 200 participants and evaluate the use of nicotinamide and lanthanum carbonate in a double-blind, placebo-controlled trial over 12 months. The primary aim of COMBINE is the safety and biochemical efficacy of these phosphate-lowering agents, although assessments of circulating biomarkers of bone and mineral metabolism and MRI of intermediate measures of cardiovascular disease will also be measured. Enrolment for this RCT began in 2015 with results expected in 2018. We have also commenced a large multicentre RCT, the IMPROVE-CKD study, to investigate the role of phosphate binders in early stages of CKD. This trial, conducted over a longer period and with a larger cohort, aims to assess potential effects of phosphate lowering on arterial compliance, vascular calcification and left ventricular mass.

\section{OVERVIEW OF THE IMPROVE-CKD STUDY \\ Study aims}

The primary objective of the IMPROVE-CKD study is to determine if use of the non-calcium-based phosphate binder lanthanum carbonate reduces the burden of cardiovascular disease in patients with CKD stages $3 \mathrm{~b}$ and 4 when compared with placebo. The primary end-point of the study is change in arterial compliance measured by PWV over a 96-week period. Secondary outcomes include change in aortic calcification and biochemical parameters of serum phosphate, PTH and FGF-23 levels.

\section{Study design, setting and participants}

The IMPROVE-CKD study is an investigator-led, prospective, double-blind, randomised placebo-controlled parallel-group trial. Figure 1 demonstrates the overall study design.

The IMPROVE-CKD study includes patients from 17 nephrology sites in Australia, New Zealand and Malaysia with CKD stages $3 \mathrm{~b}$ and 4 , aged over 18 years old and who have a serum phosphate level over $1.00 \mathrm{mmol} / \mathrm{L}$ $(3.10 \mathrm{mg} / \mathrm{dL})$ on at least one occasion in the 6 months prior to randomisation (box 1). Patient recruitment commenced in 2012, with a plan to enrol 488 participants for a 96-week study period. During the recruitment phase of the study, changes were made to the inclusion and exclusion criteria in order to improve feasibility and 


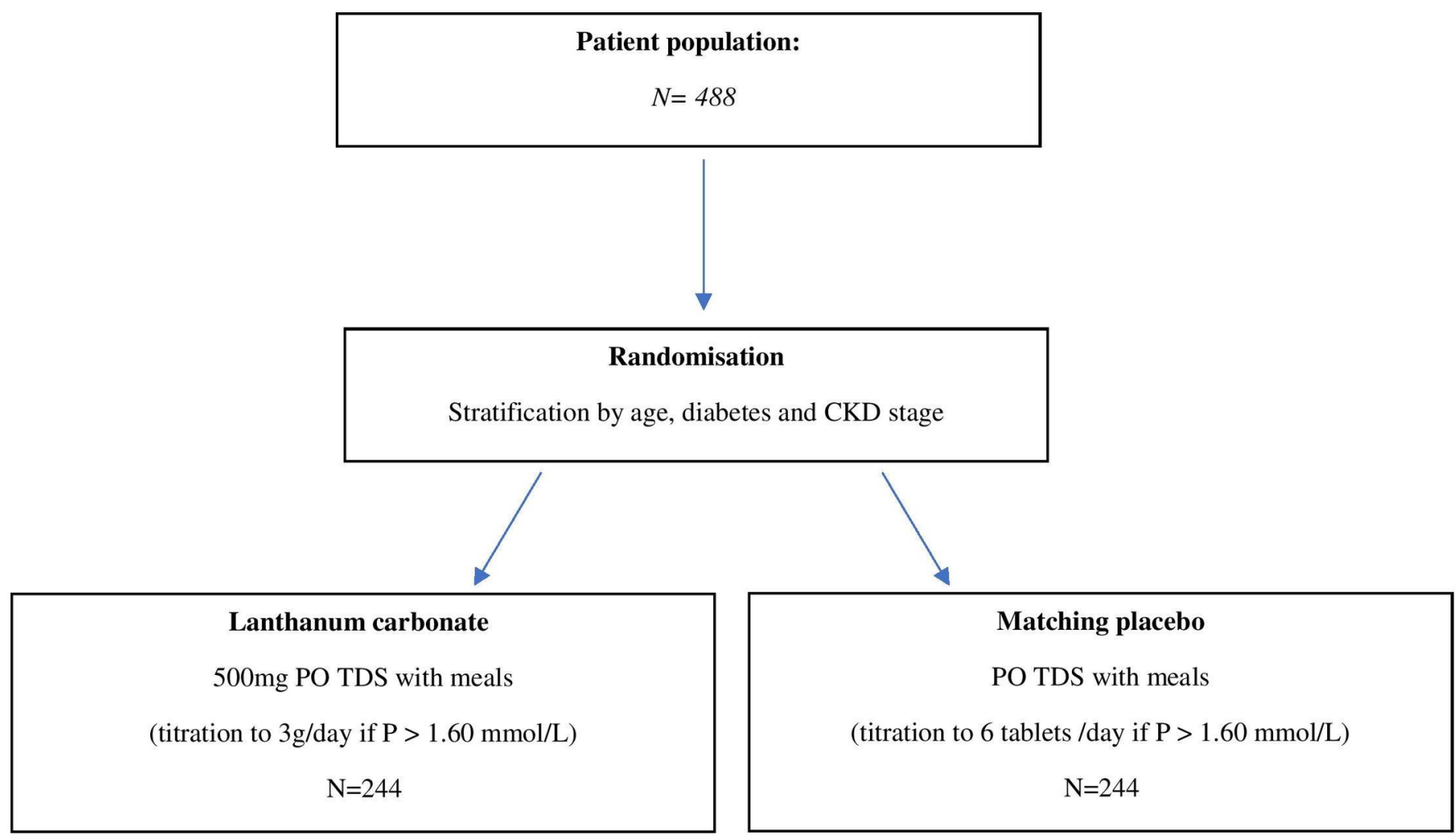

Trial outcomes at 96 weeks

Primary outcome

Arterial compliance (measured by PWV)

Key secondary outcomes

a) Aortic calcification (measured with CT)

b) Left ventricular mass (measured with MRI)

c) Serum phosphate, calcium, PTH, and FGF-23

d) Renal function (eGFR and \% change)

e) BMD at lumbar spine (measured by CT)

Figure 1 Schema of the IMPROVE-CKD trial. BMD, bone mineral density; eGFR, estimated glomerular filtration rate; FGF23, fibroblast growth factor 23; IMPROVE-CKD, Impact of Phosphate Reduction On Vascular End-points in CKD; PTH, parathyroid hormone ; PWV, pulse wave velocity.

generalisability of the trial. The changes and rationale are documented in table 2.

\section{Experimental intervention}

Lanthanum carbonate (Fosrenol) is a commercial product of Shire Pharmaceuticals for the treatment of hyperphosphatemia. ${ }^{24}$ On ingestion, lanthanum ions are released from the carbonate as a result of gastric acid secretion in the upper gastrointestinal tract and bind to dietary phosphate. The compound lanthanum phosphate is insoluble and lanthanum carbonate has been shown to effectively reduce the absorption of dietary phosphate in the CKD population. In comparison to calcium-based phosphate binders, lanthanum carbonate has additional benefits of reduced incident hypercalcemia. ${ }^{25}$ Lanthanum has been studied in both predialysis and dialysis patients, with significant reduction in serum phosphate in both patient groups.

The long-term safety of lanthanum carbonate in patients with CKD has been reported, with randomised prospective studies revealing no evidence of bone accumulation or liver toxicity, ${ }^{26}$ and after more than 850 000 person-years of worldwide patient exposure, there is no evidence that lanthanum carbonate is associated with adverse safety outcomes in CKD patients. ${ }^{26}$

\section{Treatment randomisation}

Participants are randomised 1:1 to two arms involving lanthanum carbonate $500 \mathrm{mg}$ three times daily with 


\begin{tabular}{|c|c|c|c|}
\hline $\begin{array}{l}\text { Issue and } \\
\text { version of } \\
\text { protocol }\end{array}$ & $\begin{array}{l}\text { Albuminuria/proteinuria; changes } \\
\text { between protocol versions } 1.0 \text { and } \\
2.0\end{array}$ & $\begin{array}{l}\text { Serum phosphate level; changes } \\
\text { between protocol versions } 1.0 \text { to } 2.0 \\
\text { and } 3.0\end{array}$ & $\begin{array}{l}\text { Pulse wave velocity; changes } \\
\text { between protocol versions } 2.0 \text { and } 3.0\end{array}$ \\
\hline $\begin{array}{l}\text { Protocol } \\
\text { amendment }\end{array}$ & $\begin{array}{l}\text { Initial inclusion criteria were urine } \\
\text { albumin/creatinine ratio (ACR) }>10 \mathrm{mg} / \\
\mathrm{mmol} \text { or protein/creatinine ratio } \\
(\mathrm{PCR})>15 \mathrm{mg} / \mathrm{mmol} \text {. } \\
\text { These criteria were subsequently } \\
\text { removed. }\end{array}$ & $\begin{array}{l}\text { Inclusion criterion was initially for serum } \\
\text { phosphate level }>1.2 \mathrm{mmol} / \mathrm{L}(3.72 \mathrm{mg} / \mathrm{dL}) \text { at } \\
\text { screening, but was changed to }>1.2 \mathrm{mmol} / \mathrm{L} \\
\text { on at least one occasion over the previous } \\
6 \text { months. } \\
\text { The serum phosphate level was } \\
\text { then subsequently reduced } \\
\text { from }>1.2 \text { to }>1.0 \mathrm{mmol} / \mathrm{L}(3.10 \mathrm{mg} / \mathrm{dL}) \text { on } \\
\text { at least one occasion over the previous } \\
6 \text { months. }\end{array}$ & $\begin{array}{l}\text { Exclusion criterion was added as inability } \\
\text { to obtain a PWV measure. }\end{array}$ \\
\hline $\begin{array}{l}\text { Rationale for } \\
\text { change }\end{array}$ & $\begin{array}{l}\text { The initial introduction of these criteria } \\
\text { was to increase the proportion of CKD } \\
3 \mathrm{~b} / 4 \text { subjects who were at greater risk } \\
\text { of cardiovascular disease as well as } \\
\text { increased risk of progression of renal } \\
\text { impairment. Review of screening logs } \\
\text { showed that albuminuria/proteinuria } \\
\text { were the the most common exclusion } \\
\text { criteria; therefore, these were removed } \\
\text { as they were not considered essential, } \\
\text { posed a significant impediment to } \\
\text { recruitment and the population for } \\
\text { recruitment was already deemed } \\
\text { at increased cardiovascular risk } \\
\text { regardless of albuminuria/proteinuria. } \\
\text { Also, for this study, pulse wave velocity } \\
\text { (PWV) is the primary surrogate end- } \\
\text { point and there is only one publication } \\
\text { which links albuminuria/proteinuria } \\
\text { to PWV, so this association is not } \\
\text { considered significant. }{ }^{46}\end{array}$ & $\begin{array}{l}\text { The window for serum phosphate results } \\
\text { to screen eligible patients was initially } \\
\text { changed to be within the previous } 6 \text { months } \\
\text { due to fluctuations above and below levels } \\
\text { of } 1.2 \text { mmol/L. This change was made to } \\
\text { improve study recruitment by broadening } \\
\text { the population of suitable participants. Trial } \\
\text { screening log review continued to show } \\
\text { that this inclusion criterion was a major } \\
\text { barrier to patient eligibility for the study } \\
\text { and therefore the level was reduced to } \\
1.0 \text { mmol/L. This change was not thought } \\
\text { to adversely affect the study outcomes } \\
\text { because although the study is using a } \\
\text { phosphate binder as an intervention, } \\
\text { the primary purpose of the study is the } \\
\text { attenuation of cardiovascular outcomes. } \\
\text { A lower phosphate level was added as an } \\
\text { exclusion criterion to ensure that patients } \\
\text { with very low phosphate levels are not } \\
\text { included into the study where further } \\
\text { phosphate reduction may adversely affect } \\
\text { their health. }\end{array}$ & $\begin{array}{l}\text { As the trial progressed, a small } \\
\text { percentage of the population was noted } \\
\text { on whom a PWV reading could not be } \\
\text { obtained. This was therefore added } \\
\text { as an exclusion criterion in order to } \\
\text { ensure the power of the study is not } \\
\text { compromised by including individuals } \\
\text { whose PWV is unattainable at screening. } \\
\text { It is increasingly recognised that even for } \\
\text { experienced operators, there is a small } \\
\text { percentage of the population on whom } \\
\text { PWV cannot be obtained. This has been } \\
\text { documented on other studies. }{ }^{47-49}\end{array}$ \\
\hline
\end{tabular}

IMPROVE-CKD, Impact of Phosphate Reduction On Vascular End- points in CKD.

meals or matching placebo three times daily with meals for 96 weeks. Study medication is uptitrated by local investigators to a total dose of six tablets daily $(3000 \mathrm{mg} /$ day lanthanum carbonate) if serum phosphate remains persistently greater than $1.60 \mathrm{mmol} / \mathrm{L}(4.95 \mathrm{mg} / \mathrm{dL})$. Randomisation is by a covariate-adaptive algorithm minimising imbalance across treatment arms in age, presence of diabetes, study site and CKD stage and implemented via web-based access to a central electronic randomisation system provided by The George Institute in Sydney, Australia.

\section{Study procedures}

Patients who meet the eligibility criteria and provide written informed consent undergo a baseline visit. A site screening log records the number of participants screened who are potentially eligible to participate and the reasons for ineligibility and for non-participation if eligible. Potentially eligible participants already receiving a phosphate binder at screening undergo a 2-week washout period before attending the baseline visit. Tables 3 and 4 outline the study schedule visits and laboratory outcome measures for the 96 -week trial. Pill counts will be undertaken at study visits to assess adherence to study medication.

Use of concomitant medications in this study will be as per standard of clinical care. Oral vitamin D administration, both 1,25 dihydroxy vitamin $\mathrm{D}$ (calcitriol) and nutritional vitamin D (cholecalciferol), is allowed and can be prescribed at the discretion of the treating physician. Calcitriol may be administered to study patients to treat secondary hyperparathyroidism or hypocalcemia as indicated, with a maximal dose of $0.25 \mathrm{mcg}$ (one tablet) per day.

In addition to the maximal titrated study medication, calcium, magnesium or aluminium-based phosphate binders can be prescribed for persistent hyperphosphatemia at the discretion of the local investigator (recommended for progressive or persistent serum phosphate levels $>1.60 \mathrm{mmol} / \mathrm{L}$ ); and because these are the only available phosphate-lowering agents for predialysis CKD patients in Australia, New Zealand and Malaysia, this trial will have no potential drop-ins.

The IMPROVE-CKD study is being overseen by the Trial Steering Committee and centrally coordinated 
Box 1 Inclusion and exclusion criteria for the IMPROVECKD study

\section{Inclusion criteria}

1. Patients with CKD stages $3 \mathrm{~b}$ and 4 (eGFR between 15 and $45 \mathrm{~mL}$ / $\left.\min / 1.73 \mathrm{~m}^{2}\right)^{*}$.

2. Serum phosphate $>1.00 \mathrm{mmol} / \mathrm{L}(3.10 \mathrm{mg} / \mathrm{dL})$ on at least one occasion in the previous 6 months.

3. 18 years or over.

4. Able to give informed consent.

Exclusion criteria

1. Patients with a history of psychological illness or condition which interferes with their ability to understand or comply with the requirements of the study.

2. Renal transplantation.

3. Recent (within 1 month) hospitalisation or cardiovascular event.

4. Pregnancy or breast feeding.

5. Medical conditions that impact on phosphate metabolism (apart from CKD), for example, primary hyperparathyroidism or hypoparathyroidism; previous subtotal parathyroidectomy; gastrointestinal malabsorption disorders such as Crohn's disease, ulcerative colitis, coeliac disease or severe liver dysfunction.

6. Malnutrition, defined as serum albumin $<30 \mathrm{~g} / \mathrm{L}$.

7. Serum phosphate $\leq 0.8 \mathrm{mmol} / \mathrm{L}(2.48 \mathrm{mg} / \mathrm{dL})$ at screening.

8. Atrial fibrillation as documented on ECG performed at screening.

9. Inability to perform PWV.

*eGFR determined using the CKD-Epidemiology Collaboration (CKD-EPI) equation.

CKD, chronic kidney disease ; eGFR, estimated glomerular filtration rate; IMPROVE-CKD, Impact of Phosphate Reduction On Vascular Endpoints in CKD ; PWV, pulse wave velocity.

by the Australasian Kidney Trials Network (AKTN) (online supplementary appendix). The safety of participants is overseen by an independent Data Safety Monitoring Board (DSMB, online supplementary appendix). Participants, investigators, the AKTN coordinating centre staff and outcome assessors are all blinded to the treatment assignment in the IMPROVE-CKD study. Any serious adverse event will be reported to the AKTN within 1 working day of investigators becoming aware of the event; and investigators will be required to report any adverse event leading to discontinuation of the study drug. Monitors from the AKTN will review patients' records regularly to ensure that all end-points and serious adverse events will be reported. The DSMB will assess unblinded data to safeguard participant interests and enhance the integrity of the trial.

\section{Primary outcome}

The primary outcome of the IMPROVE-CKD study is change in large arterial compliance (as measured by carotid-femoral PWV) at 96 weeks after randomisation to lanthanum carbonate or placebo. Hyperphosphatemia has been associated with reduced arterial compliance and multiple studies have reported a positive relationship between serum phosphate and PWV. ${ }^{8}{ }^{2}$ PWV has been used to measure arterial compliance and is considered to be a valid surrogate for cardiovascular morbidity and mortality. ${ }^{29}{ }^{30}$ PWV also correlates with CKD stage and increases as CKD progresses. ${ }^{31}{ }^{32}$ In the IMPROVE-CKD study, arterial compliance is being measured using a SphygmoCor device (AtCor, PWV, Westmead, Sydney, Australia) with determination of carotid-femoral PWV. Carotid-femoral PWV measures the interval between pulse waves at the carotid and femoral arteries, with higher values representing stiffer vessels. In studies of patients with CKD, mean interobserver and day-to-day reproducibility of PWV measurements have been reported to be acceptable, with differences of $0.3 \pm 3.2 \mathrm{~m} / \mathrm{s}$ and $-0.7 \pm 1.9$ $\mathrm{m} / \mathrm{s}$ in some studies. ${ }^{33}$ Pulse wave analysis is also being measured using augmentation index (AI) by the SphygmoCor device as an additional outcome. AI represents the difference between early and late systolic peaks of the systolic pulse wave contour, divided by pulse pressure (\%). All PWV and AI readings will be reviewed at a central cardiac laboratory by trained study investigators blinded to patient details and study medication.

\section{Secondary outcomes}

Vascular calcification

Abdominal aortic calcification is being determined using CT at baseline and 96 weeks in the IMPROVE-CKD study. If patients exit the study after 12 months, they will be invited to have an early exit CT scan. Aortic calcification is estimated to be present in approximately $60 \%$ of patients with CKD stages 4 and 5, and increases in those on dialysis, with progressive disease related to dialysis vintage. ${ }^{34}$ Aortic calcification is related to the presence of other vascular disease, as well as to serum phosphate and PTH levels. There is an association of aortic calcification and arterial compliance, with correlations between calcium scoring via CT and $\mathrm{PWV} .^{28} \mathrm{CT}$ images will be centrally reviewed for calcification scores by a trained radiologist and radiographer blinded to patient details and study medication.

\section{Left ventricular hypertrophy}

PWV has been associated with increased left ventricular mass and the development of diastolic dysfunction in hypertensive patients. ${ }^{55}$ However, left ventricular mass has also been associated with the development of hyperphosphatemia independent of PWV. ${ }^{7}$ In the IMPROVE-CKD study, cardiac MRI, the gold standard to evaluate the development of left ventricular hypertrophy, is being used to measure left ventricular mass on a subcohort of participants at baseline and 96 weeks. If a patient exits the study after 12 months, they will be invited to have an exit MRI. MRI images will be centrally reviewed by cardiologists trained in cardiac MRI and blinded to patient details and study medication.

\section{Biochemical parameters}

Serum phosphate, calcium and PTH are being assessed at individual sites throughout the 96-week study period. FGF-23 is being measured 6-monthly using intact and c-terminal FGF-23 assays and will be centrally analysed. 


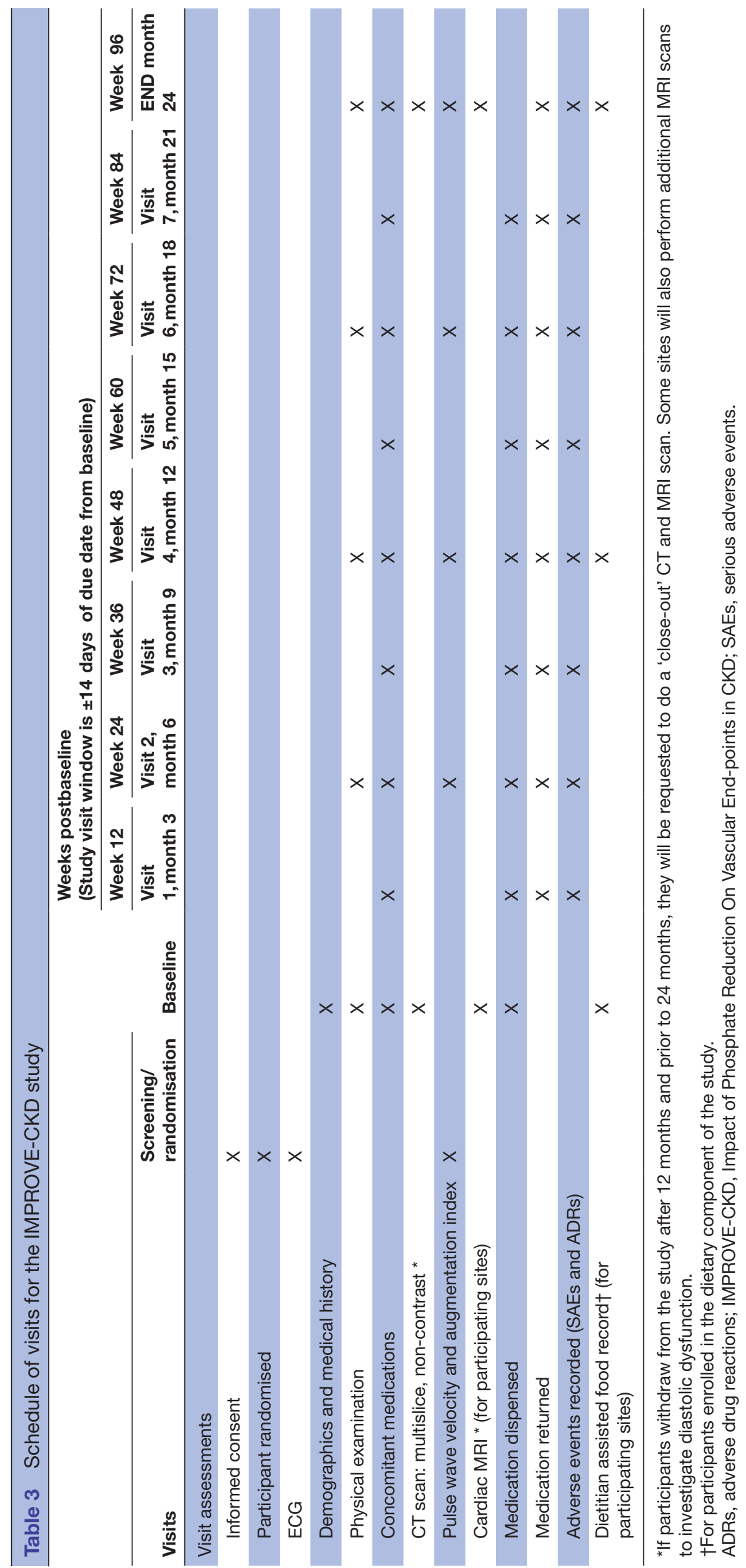


Table 4 Laboratory outcome measures in the IMPROVE-CKD study

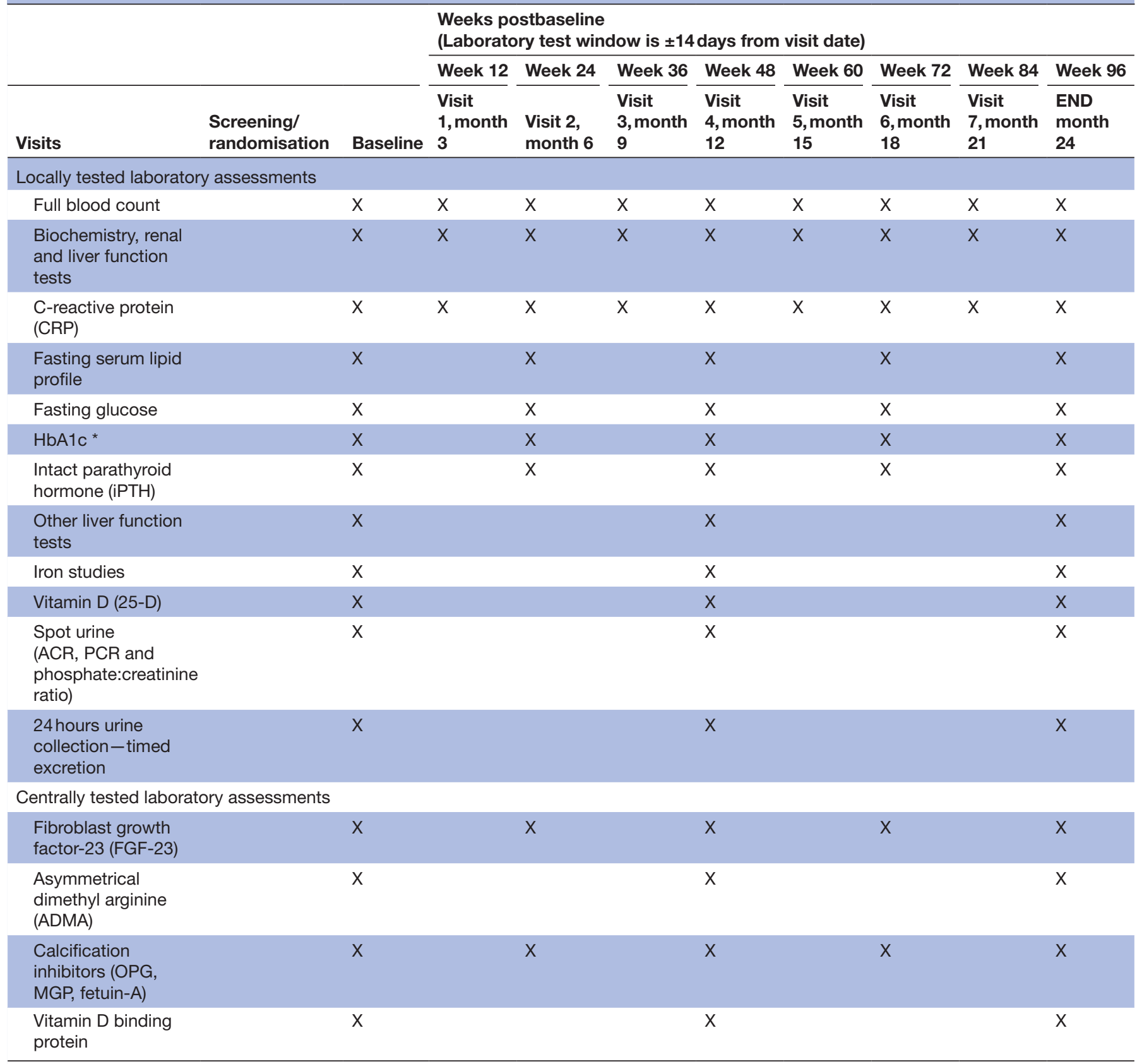

*Only performed in patients with diabetes.

ACR, albumin:creatinine ratio; IMPROVE-CKD, Impact of Phosphate Reduction On Vascular End- points in CKD; MGP, matrix Gla protein; OPG, osteoprotegerin; PCR, protein:creatinine ratio.

Changes in urinary phosphate excretion are also being evaluated using 24-hour urine collections. Standard biochemical measurements are being performed in hospital laboratories with appropriate regulatory accreditation to monitor for adverse reactions. Serum and urine samples will also be collected at several time points for exploratory analyses.

\section{Dietary phosphate}

A dietary substudy is included within IMPROVE CKD, to analyse daily dietary phosphate intake using a questionnaire at entry, 52-week and 96-week visits. Diet is the main source of exogenous phosphate, and dietary phosphate restriction is recommended in treatment algorithms prior to initiation of phosphate binder therapy. ${ }^{1}$

Bone mineral density

Bone mineral density (BMD) is also being determined from the aortic CT images at baseline and at 96 weeks. ${ }^{36}$ Due to the complexity of CKD-MBD and lack of evidencebased treatment to manage renal bone disease, BMD as determined by dual-energy X-ray absorptiometry has not been utilised routinely to predict fracture risk or monitor therapy. Low BMD, as a marker of osteoporosis and renal 
osteodystrophy, however, is a considerable component of CKD-MBD and recent prospective trials have reported that low BMD of the femoral neck and total hip in predialysis CKD patients is associated with increased fracture rates. ${ }^{37}$ Improvement in BMD has been reported with the use of phosphate binders in contrast to placebo ${ }^{21}$ and therefore BMD is an important additional outcome for the IMPROVE-CKD study.

\section{Statistical considerations \\ Sample size}

The study is designed to detect a clinically meaningful difference of $1 \mathrm{~m} / \mathrm{s}$ in PWV between the study groups at 96 weeks. Assuming a within group SD of $2.9 \mathrm{~m} / \mathrm{s}$, a sample size of 356 patients would detect a $1 \mathrm{~m} / \mathrm{s}$ difference in PWV at the 5\% significance level with $90 \%$ statistical power. To account for an estimated $10 \%$ study withdrawal rate and $10 \%$ non-adherence rate, recruitment of 488 participants is anticipated to be required. No allowance was made for drop-ins, given there is no provision for lanthanum carbonate to be accessed in the predialysis CKD population in Australia, New Zealand and Malaysia.

\section{Analysis}

Outcome data from all randomised participants will be analysed according to the intention-to-treat principle. The effect of lanthanum carbonate on PWV at 96 weeks will be estimated using analysis of covariance (ANCOVA) to adjust for baseline PWV values. Subgroup analyses will be performed according to CKD stage (3b vs 4 ), age groups ( $<60$ years, $\geq 60$ years), the presence of diabetes mellitus and levels of serum phosphate (quartiles) by examining treatment by subgroup interactions in linear regression models. Differences in other continuous variables between the lanthanum and placebo groups will be analysed by ANCOVA adjusting for baseline measurements. Differences in the percentage change in Agatston scores between the study arms are not expected to be normally distributed and will be analysed using the Wilcoxon rank sum test. Differences between treatment groups on other categorical variables will be analysed using $\mathrm{X}^{2}$ tests.

\section{Patient and public involvement}

Patients and the public were not initially involved in the development of this study protocol. However, there was a consumer representative on the AKTN Scientific Committee which reviewed the protocol, provided input and approved the study before it commenced recruitment.

\section{Ethics and dissemination}

This study is being conducted in accordance with the Declaration of Helsinki, the National Health and Medical Research Committee (NHMRC) statement on Human Experimentation, Joint NHMRC/AVCG statement and Guidelines on Practical Research, applicable International Conference on Harmonisation (ICH) guidelines and the Therapeutic Goods Administration. Results of the IMPROVE-CKD study, including exploratory outcomes, will be disseminated through publications in international peer-reviewed journals and presentations at local, national and international scientific conferences. Results will also be disseminated to all study participants in the form of a clear and detailed description of the outcomes, which will be specifically consumer-focused.

\section{DISCUSSION}

A recent systematic review and meta-analysis which evaluated evidence for correlation between the effects of CKD-MBD medications, such as phosphate binders, on biochemical parameters as well as on patient-level end-points of cardiovascular disease and mortality in patients with CKD (28 studies, 6999 participants), reported that effects on serum phosphate levels were weakly and imprecisely correlated with all-cause and cardiovascular death. ${ }^{38}$ The authors of this meta-analysis concluded that, as existing data did not exclude a mortality benefit with treatment, trials are needed to address patient-centred outcomes to evaluate drug effectiveness in CKD-MBD. ${ }^{38}$ Another recent network meta-analysis of 77 RCTs that assessed the effects of different phosphate binders on mortality, cardiovascular disease and biochemical parameters (involving $12562 \mathrm{CKD}$ patients), reported no evidence that any class of phosphate binder lowered mortality or cardiovascular events when compared with placebo. ${ }^{39}$ The vast majority of studies however involved dialysis patients (62 trials, 11009 patients) and most trials were generally of short duration (median 6 months) with high risks of bias. Again, these studies highlight the need for further RCTs involving phosphate lowering in patients with CKD not on dialysis, with longer duration of intervention and assessment of outcomes beyond changes in biochemical parameters of CKD-MBD.

The IMPROVE-CKD study, an international, multicentre RCT, plans to determine if lanthanum carbonate compared with placebo will improve or attenuate arterial compliance and reduce the progression of aortic calcification in patients with CKD. Reduced arterial compliance and increased vascular calcification are significant issues for patients with CKD, by contributing to their greater cardiovascular morbidity and mortality. At present, there are no treatment options that are proven to improve arterial stiffness or reduce vascular calcification for patients with CKD-MBD, and the cautious recommendations of international clinical guidelines reflects this lack of evidence. The IMPROVE-CKD study will provide evidence for or against the value of the phosphate binder lanthanum carbonate to affect the rate of change of aortic PWV and aortic calcification. Should this study not achieve a separation in serum phosphate values between the two arms of the trial, any differences in outcomes may reflect differences in FGF-23. FGF-23 levels at 96 weeks might be expected to differ between study arms, because FGF-23 is reported to fall with lanthanum carbonate therapy; although this relationship has not been demonstrated consistently. ${ }^{40} 41$ 
A recent open-labelled clinical trial randomised 120 patients with CKD stages 3-4 (and serum phosphate $>1.47 \mathrm{mmol} / \mathrm{L}[4.6 \mathrm{mg} / \mathrm{dL}])$ to either lanthanum carbonate, calcium acetate or dietary phosphate restriction for 12 months. ${ }^{42}$ For the 102 patients who completed the trial, assessment of biochemical parameters, including FGF-23, as well as vascular parameters of coronary artery calcification, PWV and endothelial dysfunction (as measured by reactive hyperemia index), demonstrated no difference in change of any outcome from baseline to 12 months between the three study arms, except for PTH which was suppressed more in those on calcium-based phosphate binders. Overall, there was also a mild reduction in bone-specific alkaline phosphatase between baseline and 12 months when results from all measures to reduce phosphate absorption were combined, which may potentially suggest improved bone turnover with phosphate lowering. This study however raises further concerns about the lack of proven benefits of phosphate-lowering therapy in the non-dialysis CKD population.

There is a growing understanding of circadian rhythm and the variability in relation to serum phosphate levels, with recent appreciation for the contribution of the nicotinamide phosphoribosyltransferase (Nampt)/nicotinamide adenine dinucleotide (NAD) system to the regulation of sodium-phosphate cotransporters. Nampt is a regulator of the intracellular NAD pool and this pathway is involved in renal and intestinal expression of sodium-dependent phosphate transporters. ${ }^{43}$ One of the potential reasons for the commonly observed heterogeneity in response to phosphate binders may be due to interindividual variability in transcellular phosphate movement. ${ }^{44}$ Circadian variation in serum phosphate may not be regulated by hormones but determined by the Nampt/NAD system, which affects cellular shifts and renal and intestinal phosphate transport; and the time of day serum phosphate is measured may be critical to determining treatment effect with interventions directed at regulating serum phosphate. Morning serum phosphate is now considered less helpful when assessing any change from interventions, with the best time to detect a difference in the afternoon. One crossover feeding study in patients with CKD reported the circadian pattern of serum phosphate with the lowest concentrations at 0800 hours and highest at $1600 .{ }^{45}$ Therefore, the effect of lanthanum carbonate on serum phosphate levels may or may not indicate a treatment effect; however, this contributes to the rationale for why the IMPROVE-CKD study is assessing outcome measures other than serum phosphate to assess efficacy, and these other measures will more accurately reflect the impact of modifying phosphate homeostasis.

\section{Clinical implications of the study}

If the use of lanthanum carbonate in the IMPROVE-CKD study has a beneficial effect on surrogates for cardiovascular disease, this will provide support for the current hypothesis that phosphate binders and control of hyperphosphatemia may mitigate adverse cardiovascular outcomes in patients with CKD. Although a larger trial would be required to demonstrate a benefit on mortality, the use of surrogate cardiovascular outcomes should provide clinicians with improved evidence regarding treatment strategies. Importantly, this study using lanthanum carbonate, a non-calcium-based phosphate binder, allows for a more accurate assessment of the effect of phosphate binding strategies on vascular calcification, without the complication of an exogenous calcium load. This may provide evidence for the role of non-calcium-based binders in the predialysis CKD population, and help inform CKD-MBD guidelines that currently lack strong evidence for phosphate control in CKD stages 3-5. In addition, the IMPROVE-CKD study will provide valuable information regarding the relationship between phosphate, FGF-23, arterial compliance and calcification in patients with CKD.

\section{Author affiliations}

${ }^{1}$ Department of Nephrology, Royal Melbourne Hospital, Melbourne, Victoria, Australia ${ }^{2}$ Department of Medicine, University of Melbourne, Parkville, Victoria, Australia ${ }^{3}$ Epworth Healthcare, Melbourne, Victoria, Australia

${ }^{4}$ Department of Renal Medicine, Westmead Hospital, Sydney, New South Wales, Australia

${ }^{5}$ Department of Nephrology, St. George Hospital, Sydney, New South Wales, Australia ${ }^{6}$ Faculty of Medicine, University of Queensland, Brisbane, Queensland, Australia ${ }^{7}$ Australasian Kidney Trials Network, Brisbane, Queensland, Australia ${ }^{8}$ University of Queensland, Australasian Kidney Trials Network, Brisbane, Queensland, Australia

Collaborators Full list of members for the 'IMPROVE-CKD Writing Committee': Nigel D Toussaint; Eugenia Pedagogos; Nicole Lioufas; Carmel M Hawley; Grahame J Elder; Elaine M Pascoe; Andrea Valks; Sunil V Badve; Geoffrey A Block; Neil C Boudville; Katrina Campbell; James D Cameron; Sylvia S M Chen; Randall J Faull; Stephen G Holt; Lai S Hooi; Dana Jackson; Meg J Jardine; David W Johnson; Peter G Kerr; Kenneth K Lau; Alicia Morrish; Vlado Perkovic; Kevan R Polkinghorne; Carol A Pollock; Donna Reidlinger; Laura Robison; Edward R Smith; Robert J Walker; Angela Yee Moon Wang.

Contributors All authors (NDT, EP, NL, CH, GE, EP, AV, SVB, GAB, NCB, KC, JDC, SSMC, RJF, SGH, LSH, DJ, MJJ, DWJ, PGK, KKL, AM, VP, KRP, CAP, DR, LR, ERS, RJW and AYMW) were involved in study design and concept. NDT, EP, NL, CMH, GJE, EMP, $A V$ and SVB drafted the manuscript, and all authors approved the final version of the manuscript.

Funding The IMPROVE-CKD study was funded by grants from the National Health and Medical Research Council (NHMRC) of Australia Project Grant (APP1044302); NHMRC Enabling Grant (ID 631731); NHMRC Programme Grant (APP1092957) and Shire Pharmaceuticals Development Limited Clinical Trial Contribution. Study medication (lanthanum carbonate and matching placebo) was supplied by Shire Pharmaceuticals.

Competing interests NDT has received honoraria, travel support and research funding from Amgen, Shire and Sanofi. CH has received research funding from Amgen and Shire. GJE has received honoraria, travel support and research funding from Amgen and Sanofi. SGH has received honoraria, travel support or research funding from Amgen and Sanofi. DWJ has received consultancy fees from Sanofi, travel support from Amgen and is a current recipient of an Australian National Health and Medical Research Council Practitioner Fellowship. ERS has received research funding from Amgen and Sanofi and owns stock in Calciscon.

Patient consent for publication Obtained.

Ethics approval Ethical approval for the IMPROVE-CKD trial was obtained by each local Institutional Ethics Committee for each participating site prior to study commencement.

Provenance and peer review Not commissioned; externally peer reviewed.

Open access This is an open access article distributed in accordance with the Creative Commons Attribution Non Commercial (CC BY-NC 4.0) license, which permits others to distribute, remix, adapt, build upon this work non-commercially, 
and license their derivative works on different terms, provided the original work is properly cited, appropriate credit is given, any changes made indicated, and the use is non-commercial. See: http://creativecommons.org/licenses/by-nc/4.0/.

\section{REFERENCES}

1. Kidney Disease: Improving Global Outcomes (KDIGO) CKDMBD Work Group. KDIGO clinical practice guideline for the diagnosis, evaluation, prevention, and treatment of Chronic Kidney Disease-Mineral and Bone Disorder (CKD-MBD). Kidney Int Suppl 2009:S1-130.

2. Fort J. Chronic renal failure: A cardiovascular risk factor. Kidney Int 2005;68:S25-9.

3. Hruska K, Mathew S, Lund R, et al. Cardiovascular risk factors in chronic kidney disease: does phosphate qualify? Kidney Int 2011;79:S9-13.

4. Jamal SA, Vandermeer B, Raggi P, et al. Effect of calcium-based versus non-calcium-based phosphate binders on mortality in patients with chronic kidney disease: an updated systematic review and meta-analysis. Lancet 2013;382:1268-77.

5. Patel L, Bernard LM, Elder GJ. Sevelamer versus calcium-based binders for treatment of hyperphosphatemia in CKD: a metaanalysis of randomized controlled trials. Clin J Am Soc Nephrol 2016;11:232-44.

6. Tonelli M, Sacks F, Pfeffer M, et al. Relation between serum phosphate level and cardiovascular event rate in people with coronary disease. Circulation 2005;112:2627-33.

7. Chue CD, Edwards NC, Moody WE, et al. Serum phosphate is associated with left ventricular mass in patients with chronic kidney disease: a cardiac magnetic resonance study. Heart 2012;98:219-24

8. Six I, Maizel J, Barreto FC, et al. Effects of phosphate on vascular function under normal conditions and influence of the uraemic state. Cardiovasc Res 2012;96:130-9.

9. Jüppner H. Phosphate and FGF-23. Kidney Int 2011;79:S24-S27.

10. Ozkok A, Kekik C, Karahan GE, et al. FGF-23 associated with the progression of coronary artery calcification in hemodialysis patients. BMC Nephrol 2013;14:241.

11. Isakova T, Ix JH, Sprague SM, et al. Rationale and approaches to phosphate and fibroblast growth factor 23 reduction in CKD. J Am Soc Nephrol 2015;26:2328-39.

12. Kimura K, Saika Y, Otani H, et al. Factors associated with calcification of the abdominal aorta in hemodialysis patients. Kidney Int Suppl 1999;71:S238-41.

13. Moe SM, Chen NX. Mechanisms of vascular calcification in chronic kidney disease. J Am Soc Nephrol 2008;19:213-6.

14. Nasrallah MM, El-Shehaby AR, Salem MM, et al. Fibroblast growth factor-23 (FGF-23) is independently correlated to aortic calcification in haemodialysis patients. Nephrol Dial Transplant 2010;25:2679-85.

15. Scialla JJ, Lau WL, Reilly MP, et al. Fibroblast growth factor 23 is not associated with and does not induce arterial calcification. Kidney Int 2013;83:1159-68.

16. Habbous S, Przech S, Acedillo R, et al. The efficacy and safety of sevelamer and lanthanum versus calcium-containing and iron-based binders in treating hyperphosphatemia in patients with chronic kidney disease: a systematic review and meta-analysis. Nephrol Dial Transplant 2017;32:111-25

17. Kdigokc-Muwg. K. clinical practice guideline update for the diagnosis, evaluation, prevention, and treatment of Chronic Kidney Disease-Mineral and Bone Disorder (CKD-MBD). Kidney Int Suppl 2017:1-59.

18. Lopes AA, Tong L, Thumma J, et al. Phosphate binder use and mortality among hemodialysis patients in the Dialysis Outcomes and Practice Patterns Study (DOPPS): evaluation of possible confounding by nutritional status. Am J Kidney Dis 2012;60:90-101.

19. Isakova T, Gutiérrez OM, Chang Y, et al. Phosphorus binders and survival on hemodialysis. J Am Soc Nephrol 2009;20:388-96

20. Fernández-Martín JL, Carrero JJ, Benedik M, et al. COSMOS: the dialysis scenario of CKD-MBD in Europe. Nephrol Dial Transplant 2013;28:1922-35.

21. Block GA, Wheeler DC, Persky MS, et al. Effects of phosphate binders in moderate CKD. J Am Soc Nephrol 2012;23:1407-15.

22. Chue CD, Townend JN, Moody WE, et al. Cardiovascular effects of sevelamer in stage 3 CKD. J Am Soc Nephrol 2013;24:842-52.

23. Seifert ME, de las Fuentes $L$, Rothstein M, et al. Effects of phosphate binder therapy on vascular stiffness in early-stage chronic kidney disease. Am J Nephrol 2013;38:158-67.

24. Swainston Harrison T, Scott LJ. Lanthanum carbonate. Drugs 2004;64:985-96.
25. Zhang C, Wen J, Li Z, et al. Efficacy and safety of lanthanum carbonate on chronic kidney disease-mineral and bone disorder in dialysis patients: a systematic review. BMC Nephrol 2013;14:226.

26. Hutchison AJ, Wilson RJ, Garafola S, et al. Lanthanum carbonate: safety data after 10years. Nephrology 2016;21:987-94.

27. Zhai CJ, Yang XW, Sun J, et al. Efficacy and safety of lanthanum carbonate versus calcium-based phosphate binders in patients with chronic kidney disease: a systematic review and meta-analysis. Int Urol Nephrol 2015;47:527-35.

28. Toussaint ND, Lau KK, Strauss BJ, et al. Associations between vascular calcification, arterial stiffness and bone mineral density in chronic kidney disease. Nephrol Dial Transplant 2008;23:586-93.

29. Pannier B, Guérin AP, Marchais SJ, et al. Stiffness of capacitive and conduit arteries. Hypertension 2005;45:592-6.

30. Blacher J, Guerin AP, Pannier B, et al. Impact of aortic stiffness on survival in end-stage renal disease. Circulation 1999;99:2434-9.

31. Tholen S, Klofat K, Pan CR, et al. Progression of aortic pulse wave velocity in patients with chronic kidney disease. J Clin Hypertens 2013;15:833-8.

32. Townsend RR, Anderson AH, Chirinos JA, et al. Association of pulse wave velocity with chronic kidney disease progression and mortality: findings from the CRIC study (chronic renal insufficiency cohort). Hypertension 2018;71:1101-7.

33. Frimodt-Møller M, Nielsen AH, Kamper AL, et al. Reproducibility of pulse-wave analysis and pulse-wave velocity determination in chronic kidney disease. Nephrol Dial Transplant 2008;23:594-600.

34. Raggi P, Boulay A, Chasan-Taber S, et al. Cardiac calcification in adult hemodialysis patients. A link between end-stage renal disease and cardiovascular disease? J Am Coll Cardiol 2002;39:695-701.

35. Brandts A, van Elderen SG, Westenberg JJ, et al. Association of aortic arch pulse wave velocity with left ventricular mass and lacunar brain infarcts in hypertensive patients: assessment with MR imaging. Radiology 2009;253:681-8.

36. Lee SJ, Binkley N, Lubner MG, et al. Opportunistic screening for osteoporosis using the sagittal reconstruction from routine abdominal CT for combined assessment of vertebral fractures and density. Osteoporos Int 2016;27:1131-6.

37. Yenchek RH, Ix JH, Shlipak MG, et al. Bone mineral density and fracture risk in older individuals with CKD. Clin J Am Soc Nephrol 2012;7:1130-6.

38. Palmer SC, Teixeira-Pinto A, Saglimbene V, et al. Association of drug effects on serum parathyroid hormone, phosphorus, and calcium levels with mortality in CKD: a meta-analysis. Am J Kidney Dis 2015;66:962-71.

39. Palmer SC, Gardner S, Tonelli M, et al. Phosphate-Binding Agents in Adults With CKD: a network meta-analysis of randomized trials. Am J Kidney Dis 2016;68:691-702.

40. Gonzalez-Parra E, Gonzalez-Casaus ML, Galán A, et al. Lanthanum carbonate reduces FGF23 in chronic kidney disease Stage 3 patients. Nephrol Dial Transplant 2011;26:2567-71.

41. Isakova T, Barchi-Chung A, Enfield G, et al. Effects of dietary phosphate restriction and phosphate binders on FGF23 levels in CKD. Clin J Am Soc Nephrol 2013;8:1009-18.

42. Kovesdy CP, Lu JL, Wall BM, et al. Changes with lanthanum carbonate, calcium acetate, and phosphorus restriction in CKD: a randomized controlled trial. Kidney Int Rep 2018;3:897-904.

43. Miyagawa A, Tatsumi S, Takahama W, et al. The sodium phosphate cotransporter family and nicotinamide phosphoribosyltransferase contribute to the daily oscillation of plasma inorganic phosphate concentration. Kidney Int 2018;93:1073-85.

44. Isakova T, Block $\mathrm{G}$. The phosphate bucket list. Kidney Int 2018;93:1033-5

45. Ix JH, Anderson CA, Smits $\mathrm{G}$, et al. Effect of dietary phosphate intake on the circadian rhythm of serum phosphate concentrations in chronic kidney disease: a crossover study. Am J Clin Nutr 2014;100:1392-7.

46. Mclntyre NJ, Fluck RJ, Mclntyre CW, et al. Determinants of arterial stiffness in chronic kidney disease stage 3. PLoS One 2013:8:e55444.

47. Dart AM, Gatzka CD, Cameron JD, et al. Large artery stiffness is not related to plasma cholesterol in older subjects with hypertension. Arterioscler Thromb Vasc Biol 2004;24:962-8.

48. Mitchell GF, Hwang SJ, Vasan RS, et al. Arterial stiffness and cardiovascular events: the framingham heart study. Circulation 2010;121:505-11.

49. Dart AM, Cameron JD, Gatzka CD, et al. Similar effects of treatment on central and brachial blood pressures in older hypertensive subjects in the second australian national blood pressure trial. Hypertension 2007;49:1242-7. 